\title{
PACIENTES POLICONSULTANTES: ¿UN SÍNTOMA DEL SISTEMA DE SALUD EN CHILE?
}

\author{
Gonzalo Miranda Hiriart ${ }^{1}$, Ximena Saffie Gatica ${ }^{2}$
}

Resumen: En el presente artículo se exponen una serie de reflexiones producto de un estudio exploratorio y cualitativo realizado en un Centro de Salud Familiar de Santiago de Chile. La pregunta que guía este artículo es si se puede considerar la alta tasa de pacientes policonsultantes como un síntoma de que algo no funciona en el sistema público de salud chileno. Se concluye que existe un desencuentro entre la demanda del paciente y el saber de los profesionales, el cual deja en evidencia la falta de claves de la medicina biológica para interpretar este tipo de quejas. Dada la ausencia de respuesta, estos pacientes suelen ser derivados a las "duplas psicosociales", sin mucha claridad sobre los fundamentos de esta derivación, dando cuenta así de algunas insuficiencias de un sistema sanitario que dice operar bajo un paradigma "biopsicosocial". Esto se ve agravado por la forma de gestionar la atención sanitaria impuesta en las últimas dos décadas, pues se trata de pacientes que atentan contra la eficiencia de las instituciones y no caben en las metas establecidas en torno a diagnósticos. Sin embargo, mientras el sistema de salud busca veladamente deshacerse de dichos pacientes, estos más insisten con sus demandas.

Palabras clave: policonsulta, sistema de salud, biomedicina, enfoque biopsicosocial

\section{Poly-consultant patients: A symptom of the Chilean health care system?}

\begin{abstract}
This paper shows a series of reflections resulting from an exploratory and qualitative study carried out in a Family Health Care Center of Santiago, Chile. The question guiding this paper consists in considering whether the high rate of poly-consultant patients is a symptom of malfunctioning of the public Chilean health care system. It is concluded that there is a discrepancy between patient demands and professional knowledge, evidencing lack of biomedical keys to interpret this type of demands. Given de absence of answers, these patients generally are derived to "psychosocial couples", without much clarity over the basis of this derivation; thus, accounting for some deficiencies of a health care system which pretends to operate under a "bio-psychosocial" paradigm. This is aggravated by the way the managing of health care is imposed in the last two decades, since these are patients which face the efficiency of institutions and they do not enter into the established goals assigned to the different diagnosis. Nevertheless, while the health care system seeks in a veiled way to get rid of these patients, these insist even more with their demands.
\end{abstract}

Key words: poly-consulting, health care system, biomedicine, bio-psychosocial focus

Pacientes policonsultantes: um sintoma do sistema de saúde no Chile?

Resumo: No presente artigo se expóe uma série de reflexôes produto de um estudo exploratório e qualitativo realizado num Centro de Saúde Familiar de Santiago do Chile. A pergunta que guia este artigo é se pode ser considerada a alta taxa de pacientes policonsultantes como um sintoma de que algo náo funciona no sistema público de saúde chileno. Conclui-se que existe um desencontro entre a demanda do paciente e o saber dos profissionais, que deixa em evidência a falta de chaves da medicina biológica para interpretar este tipo de queixas. Dada a ausência de resposta, estes pacientes podem ser derivados para as "duplas psicossociais", sem muita clareza sobre os fundamentos desta derivação, dando conta assim de algumas insuficiências de um sistema sanitário que diz operar sob um paradigma "biopsicossocial". Isto se vê agravado pela forma de gerir a atençáo sanitária imposta nas últimas duas décadas, pois se trata de pacientes que atentam contra a eficiência das instituições e náo cabem nas metas estabelecidas em torno de diagnósticos. No entanto, enquanto o sistema de saúde busca veladamente desfazer-se destes pacientes, eles mais insistem com suas demandas.

Palavras-chave: policonsulta, sistema de saúde, biomedicina, enfoque biopsicossocial

\footnotetext{
${ }^{1}$ Departamento de Psicología, Universidad Católica Silva Henríquez, Santiago, Chile Correspondencia: gmiranda@ucsh.cl

${ }^{2}$ Child Trends, Maryland, United States
} 


\section{Introducción}

Aun cuando el término "síntoma" tiende a desaparecer de los diccionarios especializados, algunos medios de divulgación de los conceptos médicos aún rescatan su valor. He aquí un ejemplo interesante: "cuando usted está enfermo, por lo general tienen síntomas — cambios que le permiten saber que algo no está bien. Al relatar a un médico sus síntomas, usted puede ayudarlo a averiguar lo que está mal. Piense en los síntomas como pistas que necesita para resolver un misterio"(1). Es en ese sentido que hablaremos de la policonsulta como un posible síntoma del sistema sanitario; es decir, en tanto fenómeno que hace visible algo que no estaría bien en el sistema.

Si bien no hay una definición clara de lo que se entiende por "paciente policonsultante", existe cierto consenso en que dicha denominación responde a pacientes que presentan un elevado número de consultas espontáneas - más de siete en un año- en un mismo centro de salud $(2,3)$. Lo que resulta más relevante es que dichas consultas aparecen como injustificadas para los profesionales que las reciben. Esto último debido a que los pacientes refieren síntomas múltiples y recurrentes, tales como náuseas, vómitos, cefaleas, e incluso síntomas que, agrupados, constituirían síndromes funcionales, como fibromialgia o colon irritable; pero ni las causas ni el curso que sigue dicha sintomatología se ajusta a los requisitos para ser consideradas "enfermedades" (4). Como hipótesis, se suele suponer que se trata de depresiones encubiertas, de trastornos somatomorfos o, simplemente, de intentos de manipulación. Además, suelen ser pacientes poco dóciles, que muestran cierta resistencia e inadecuación a los protocolos de tratamiento(5).

La alta presencia de este fenómeno en el ámbito público - se estima que entre un 10 a un $30 \%$ del total de consultas de Atención Primaria en Chile corresponde a este tipo de pacientes(6) - ha llevado a que se constituya como un problema prioritario en salud pública, ya que su existencia atenta directamente contra el uso eficiente de los recursos. Al mismo tiempo, la policonsulta sitúa a los profesionales de la salud en una situación incómoda, en tanto pone en jaque el modo de diagnosticar y tratar usado por la medicina contemporánea.
Ciertamente, los policonsultantes viajan por los distintos niveles del sistema y son estudiados a través de exámenes de laboratorio, radiografías, escaner, etc., intentando encontrar el origen de su padecer. Sin embargo, las más de las veces se hace imposible encontrar alguna evidencia que permita justificar las quejas. Tanto las técnicas diagnósticas como el examen clínico suelen mostrar que estos pacientes "no tienen nada":

"A veces, la placa que hace visible el cuerpo le muestra al médico que su enfermo 'no tiene nada', contrariamente a lo que sostiene este último que se queja de varias molestias. La oposición entre la enfermedad del médico y la del enfermo es, entonces, clara. La prueba objetiva del mal no fue proporcionada (...), de modo que el sufrimiento es imputado a la fantasía enfermiza del paciente. Es un enfermo imaginario" (7:208-209).

Los pacientes retornan a la Atención Primaria, donde son recibidos con actitudes ambivalentes e incluso agresivas, llegando en ocasiones al extremo que los profesionales se niegan a seguir atendiéndolos(8). Así, los policonsultantes reciben del sistema una descalificación de sus molestias, un menosprecio de su queja, lo que puede llegar a ser incluso más desconcertante que un diagnóstico doloroso:

"Si afirmaciones como 'usted no tiene nada', o 'esto es funcional', o 'esto es psicológico' puede durante un tiempo dar seguridad al médico (y desembarazarlo de los enfermos molestos), apenas dan tanta seguridad al enfermo. Pues en efecto se trata de 'cualquier cosa' o, peor aún, de 'nada'. Pero, ¿no es acaso esa 'nada' la que me hace sufrir, y la que nuestra cultura no nos ha enseñado a comprender, ni como médico ni como enfermo?(9:284).

A pesar de ser un fenómeno cotidiano en los servicios de salud, la reflexión y el análisis que se hace sobre los denominados policonsultantes es escasa. Son más bien una molestia que se busca mantener dentro de ciertos márgenes, para que no interfieran demasiado el "funcionamiento normal" de las instituciones. Así, son una especie de síntoma que no quiere ser escuchado, que no provoca la curiosidad que debería. Las distintas formas de descalificación de dichos pacientes dis- 
minuyen las posibilidades de una aproximación comprensiva, tanto del sufrimiento que los convierte en tal, como de debilidades que evidencia el sistema sanitario al respecto.

\section{Métodos}

Las reflexiones que a continuación se exponen son el resultado de un estudio exploratorio que se realizó utilizando metodología cualitativa, durante el primer semestre de 2011 en un Centro de Salud Familiar (CESFAM) de Santiago.

Para los fines de la muestra, la noción de "pacientes policonsultantes" fue definida operacionalmente como aquellos sujetos mayores de 18 años que hubiesen presentando más de 7 consultas espontáneas durante 2010. Otro requisito fue que el paciente tuviera alguno de los siguientes diagnósticos en su historial médico: "ansiedad", "depresión" y/o "trastorno adaptativo". A partir de estos criterios se realizó una revisión de las fichas clínicas, obteniéndose 206 sujetos que se ajustaron a los requisitos. Luego se elaboró una lista al azar, procediéndose a intentar contactar por orden sucesivo a los pacientes para programar encuentros. Finalmente, y utilizando el principio de saturación teórica, se realizaron 5 entrevistas individuales en profundidad, semiestructuradas, todas a mujeres de entre 35 y 55 años.

Por otra parte, se realizaron dos grupos de discusión con "funcionarios" (profesionales de la salud que trabajan en atención directa por más de 6 meses en el CESFAM), cada uno de tres integrantes. El primero estuvo constituido por un médico, un kinesiólogo y una enfermera, y el segundo por una matrona, un psicólogo y una nutricionista.

El material obtenido con ambas técnicas fue transcrito, con el fin de realizar un análisis de contenido, utilizando para ello como referencia la Teoría Fundada.

\section{Resultados}

\section{Oferta y demanda desencontradas}

Una cuestión interesante es que los llamados policonsultantes radican su malestar en el cuerpo, es decir, se quejan de molestias físicas. Por esto, para ellos adquiere gran relevancia el hecho de ser examinados, auscultados, palpados. Esto es más relevante, incluso, que el tiempo que dure la consulta, y contrasta con la desestimación del cuerpo que hace el médico, al considerar que las molestias somáticas no son sino "excusas"(8). Sin embargo, los pacientes se resisten a que se les envíe a un profesional de salud mental; insisten en que se profundice la pesquisa orgánica, lo que genera tensión y deteriora la relación médicopaciente(10).

Otro asunto interesante es que este tipo de pacientes busca permanentemente un vínculo cercano con los profesionales, privilegiando ser atendidos por quienes los tratan por su nombre y de manera acogedora, quienes se interesan por su vida(8). De hecho, esperan poder conversar de otras cosas con ellos, más allá de la enfermedad(11). Sin embargo, esto suele no ser valorado por los funcionarios; más aún, es considerado como algo que excede a la relación profesional. Al no advertir la demanda de singularidad que hay tras la insistencia de ser atendidos por determinadas personas, los funcionarios se sienten molestos, interpretando sus conductas como una manipulación o como un intento por obtener beneficios. Asimismo, la familiaridad se les hace incómoda. La institución, al no comprender la relevancia que los pacientes atribuyen al hecho de ser atendidos por ciertos profesionales, reacciona mal, experimentando incluso esta preferencia como una amenaza, por lo que se refuerza la tendencia a alejar a este tipo de pacientes.

En las políticas de salud, todo está diseñado para que las intervenciones sean anónimas y los profesionales intercambiables. Esto representa un aspecto clave para entender el desencuentro que se produce entre los pacientes policonsultantes y el sistema sanitario, ya que no permite advertir que, además de la demanda experta - o entremezclada con esta-, existe también una demanda intersubjetiva, en la que los pacientes buscan sentirse reconocidos por los profesionales, lo que excede una respuesta netamente técnica. Sin embargo, esta demanda se encuentra con el movimiento inverso. El médico asume que el paciente "no tiene nada” y que su queja remite a algo "psicosocial”, por lo que es derivado a psicólogos y/o asistentes sociales, con la recomendación de fortalecer sus redes externas, con el fin de que la institución de 
salud no se constituya en su soporte principal.

Los funcionarios buscan alejarlos del centro de salud, mientras los pacientes buscan alivio a través de la cercanía con la institución, y especialmente con aquellos profesionales que resultan más significativos. Interpretar su conducta como manipulación o desde las "necesidades psicosociales", excluye un cuestionamiento acerca del lugar simbólico que podría estar ocupando la institución para los pacientes policonsultantes. En efecto, las respuestas que se ofrecen son de un nivel bastante concreto; se les da la licencia, el fármaco, la interconsulta o la orden para el examen, pero esto no termina con la consulta reiterada de estos pacientes. Si se analizaran las constantes demandas desde un nivel simbólico, estas adquirirían otra significación, ya que, por ejemplo, la solicitud por obtener medicamentos podría estar dando cuenta de una forma de subsidio a la ausencia de relación que se da con los funcionarios. En este sentido, se puede hipotetizar que "el medicamento toma el lugar de la escucha o del contacto que no tiene el tiempo suficiente como para satisfacer al paciente, para reconocerlo en su sufrimiento. La dimensión simbólica se restaura, de contrabando, por medio de este recurso" (7:188).

\section{¿Cómo se constituye el problema?}

La biomedicina y la "verdadera" enfermedad.

La medicina moderna adscribe al paradigma de las ciencias de la naturaleza; más aún, reduce progresivamente su arte a una tecnología que no es otra cosa que física y química aplicadas. La noción mecánica del cuerpo humano le permitió a la medicina poscartesiana grandes innovaciones, eliminando de paso la concepción que sostenía al alma como el principio de vida. A partir de Descartes, "el cuerpo ya no puede pensarse como un lugar de fuerzas en equilibrio, sino que como una máquina en movimiento"(12:162). La enfermedad, por su parte, comenzó a ser considerada una entidad neutral y objetiva, posible de ser estudiada a través de un método que conservara la misma objetividad.

Sin embargo, la medicina no alcanzó el reconocimiento científico sino hasta fines del siglo XVIII, cuando "los médicos describieron lo que, durante siglos, había permanecido por debajo del umbral de lo visible y de lo enunciable"(13:5). Este es el momento en el que se produce la superposición del espacio de configuración de la enfermedad con el del mal en el cuerpo, movimiento que fue posible a partir de los avances de la anatomía patológica. Se convierte de esta manera a la enfermedad en un "ser discernible, aislable, clasificable, cuya etiopatogenia se hace visible y faculta al médico a predecir su evolución"(14:245). La anatomía patológica funda un nuevo método de exploración, ya que las autopsias permitían al médico detenerse en las anomalías de los órganos, para establecer relaciones entre estos y los síntomas de la enfermedad. Ello resultó en que los órganos comenzaron a ser estudiados como entidades autónomas, produciéndose así una disociación definitiva entre el cuerpo y el sujeto. A partir de dicho ejercicio se modificó la relación médicopaciente, convirtiéndose de manera progresiva en una relación entre el médico y la enfermedad. De este modo, el enfermo se transforma "no en el heredero de la historia singular de un hombre determinado en determinado espacio y tiempo, sino en la falla impersonal de una función o de un órgano indiferente al hombre, al menos en sus repercusiones" (7:198). De hecho, se hace posible estar enfermo sin sentirlo, y se puede sentir enfermo sin estarlo.

El paso del siglo XIX al XX continúa la creciente biologización de la patología, en la medida en que el desarrollo científico favoreció la implementación de nuevas técnicas diagnósticas que permitían acceder con mayor precisión al cuerpo enfermo. Esto consolidó el repliegue del paciente a un lugar cada vez más secundario respecto de su patología, ya que las ganancias técnicas significaron una desvalorización del discurso del sujeto. Dicha situación se instala en la práctica, ya que hasta el siglo XIX el médico requería de la palabra del paciente para obtener la información necesaria para realizar su diagnóstico, y secundariamente lo complementaba con la observación médica; de manera que "el médico podía ser ciego, pero ciertamente no sordo" (9:287). Sin embargo, en el siglo XX la práctica se invierte, siendo cada vez más central la búsqueda de signos objetivos ojalá a través de exámenes de laboratorio- el eje del diagnóstico. 
El hecho patológico, devenido autónomo del sujeto, se convierte en una categoría ontológica, y la nosología aspira a reflejar la esencia de las enfermedades, de manera tal que la definición baste para determinar su etiología, curso, pronóstico y, ojalá, el tratamiento de elección de la manera más pura posible, eliminando toda variable subjetiva que pudiera interferir. De este modo, las peculiaridades del paciente se tornan en una molestia para la clínica, y aquellos casos difícilmente clasificables tienden a quedar fuera del ámbito médico. La medicina orgánica, al nombrar, clasificar y organizar las diferentes enfermedades, favorece una normalización del cuerpo, cuya consecuencia es la segregación de toda persona que no se ajusta a sus criterios(15).

\section{La gestión de la clínica en las instituciones pú- blicas}

Hacia fines del siglo XX se produce una alianza implícita entre las aspiraciones de la medicina biológica y un nuevo enfoque de gestión de la cosa pública. En efecto, junto a la falta de un diagnóstico preciso, el funcionamiento mismo de las instituciones de salud es un factor que participa directamente en la discriminación del paciente policonsultante.

La manera en que se ha ido estructurando el quehacer en salud pública tiene directa relación con el tipo de gestión que se ha priorizado en el ámbito gubernamental. Especialmente relevantes son los intentos realizados en Chile tras el regreso de la democracia para recuperar la capacidad del sistema público(16). Los gobiernos de la Concertación de Partidos por la Democracia, siguiendo las directrices del Banco Mundial, pusieron un fuerte acento en la gestión de los servicios públicos. Esto se vio reflejado en una serie de reformas, como la llevada a cabo el año 1995, en la cual —en el marco de la formulación presupuestaria- el Ministerio de Hacienda solicitó por vez primera que las instituciones de salud establecieran indicadores de gestión. Se exigió, además, que los recursos solicitados se respaldaran con la implementación de proyectos concretos que apuntaran a cumplir con objetivos cuantificables, y se comenzó a crear sistemas de información para evaluar la eficacia de los servicios(17).
Esto fue el comienzo de una forma de administrar la atención de salud que se continúa profundizando hasta hoy; se comenzaron a vincular las remuneraciones con los desempeños, estableciendo estímulos y recompensas propias de una gestión que se orienta a resultados; se establecieron metas de cobertura, prestaciones, tiempos de espera, y eficiencia - entendida como el ahorro en insumos-, además de introducir metas de competitividad y generación de recursos propios. Así se llega al establecimiento de "compromisos de gestión”, que se realizan entre el Ministerio de Salud y las direcciones de los Servicios. Se incluyen como parte de este acuerdo la descripción del tipo de prestaciones que se van a ofrecer, la asignación de recursos, las metas de crecimiento y los indicadores de desempeño destinados a evaluar las metas propuestas, los cuales, por lo general, son índices de actividad(17). Ahora bien, lo más relevante es que los Servicios, al alinearse con metas de salud pública, empiezan a privilegiar la oferta de determinadas prestaciones a grupos establecidos de antemano, por sobre las demandas de la población.

La medición del rendimiento por medio de metas, se traduce finalmente en que los profesionales deben ingresar un determinado número de aquellas patologías que se han establecido como objetivos sanitarios. Si se considera que el cumplimiento de dichas metas está asociado a la existencia de incentivos monetarios - tanto para el establecimiento como para los funcionarios-, es evidente que la prioridad será el número de casos que se atienden por sobre la calidad de atención que se entrega. Lo que resulta igual o más problemático es que se privilegia la obtención de un diagnóstico como objetivo en sí mismo - por sobre la comprensión del paciente en su particularidad-, diagnóstico que suele verse forzado con fines estadísticos.

De este modo, el énfasis en la gestión que caracteriza al actual sistema de salud pública contribuye a acrecentar la perturbación que experimentan los funcionarios ante los pacientes policonsultantes, quienes atentan directamente contra la eficiencia y resolutividad que se espera de ellos. En tal sentido, son pacientes que representan una amenaza para los indicadores, debido a la imposibilidad de categorizarlos de manera concluyente y a la cantidad de horas "sin sentido" que solicitan. 


\section{El recurso a "lo psicosocial"}

Junto con el desarrollo del paradigma biomédico, se han ido elaborando otros modelos que han intentado constituirse como una alternativa a este. Tal es el caso del "enfoque biopsicosocial", término que, si bien está incluido en la definición de salud de la OMS, cobró fuerza en los años setenta, cuando George Engel lo presentó como una alternativa al modelo biomédico dominante. La mención a lo biopsicosocial respondía al interés de desarrollar comprensiones holísticas, que reconocieran la importancia que adquieren las características particulares de los sujetos y su situación al momento de entender el origen y la evolución de las patologías. De esta manera, se buscaba reivindicar la palabra del paciente, para así sacarlo del lugar de objeto en el que fue ubicado por el ideal cientificista del modelo médico y resituarlo como sujeto del acto clínico(18).

En Chile, las políticas sanitarias de las últimas décadas dicen adscribir al modelo biopsicosocial. Entre las medidas que se han llevado a cabo para dar forma a este propósito, se encuentra la transformación de los consultorios en Centros de Salud Familiar (CESFAM). Siguiendo esta lógica es que en dichos centros se ha intentado implementar una modalidad de atención que incorpore el contexto de quien consulta, considerando la calidad de vida, además del entorno familiar y social del paciente: "la transformación de los consultorios a CESFAM involucra de manera prioritaria el cambio desde un paradigma centrado en la enfermedad a uno centrado en la persona y su contexto biopsicosocial"(19:1).

A pesar de la declaración del Estado respecto de la implementación de medidas sanitarias que favorezcan una mirada integral del ser humano, la verdadera política refuerza la mantención del modelo biomédico que se intenta superar. Tal es el caso del sistema de garantías explícitas (GES), asociadas a determinados diagnósticos y grupos. La selección de las patologías para ser incluidas en el GES responde a una lógica médica tradicional —además de criterios morales utilitaristas-, distinguiendo entre las patologías de salud mental, cardiovasculares y respiratorias, por ejemplo, lo cual no solo favorece la disociación entre mente y cuerpo, sino que incluso realiza una fragmen- tación del ser humano en sus distintos órganos, atentando directamente contra la comprensión holística del sujeto(20). Lo anterior representa un esfuerzo de estandarización en el cual convergen la concepción biomédica de la enfermedad con la gestión pública contemporánea. El acto médico por excelencia no es ya el cuidado del enfermo sino el diagnóstico. Esta normalización, no solo de la enfermedad sino también de los sujetos, se refuerza en las guías clínicas y los protocolos de atención que, además de los criterios de inclusión de las patologías, ofrecen también las directrices que se deben seguir en cada caso. Es decir, se define el tratamiento de antemano por sobre la necesidad particular de cada paciente. Se trata así de una respuesta para todos igual: todos organismos sin biografía ni circunstancias(21).

Sin embargo, lo "biopsicosocial" o, más específicamente, el aspecto "psicosocial" del padecer humano - pues en la práctica funciona completamente aparte de lo "bio"-, aflora con fuerza en los centros de salud cuando se habla de los pacientes denominados policonsultantes. La falta de un diagnóstico preciso, de una enfermedad reconocible, lleva a que profesionales como médicos, matronas, enfermeras y nutricionistas sientan que hacerse cargo de estos pacientes no es algo que les compete a ellos sino a las "duplas psicosociales" (psicólogo-asistente social). De esta manera, lo "psicosocial" se transforma en el depósito de lo inexplicable y obstruye una pregunta acerca de la complejidad que subyace a la conducta de los policonsultantes, cerrando de paso la posibilidad de una verdadera aproximación psicosomática hacia el dolor, el malestar y la enfermedad.

Dada la vaguedad con que se maneja el término "psicosocial" - puede ser cualquier cosa que no pertenezca en estricto rigor al plano biológico-, es poco lo que aporta a la comprensión de la policonsulta. Muy por el contrario, aparece como un término que tranquiliza la incertidumbre de los funcionarios cuando su saber es puesto en jaque y una solución fácil en términos administrativos. Por esto, más que complejizar y abrir la pregunta por los procesos psíquicos que subyacen al actuar de estos sujetos, lo psicosocial se convierte en un significante cerrado - y vacío- que se explica por sí mismo. 
Ahora bien, si se profundiza en la noción de lo psicosocial que maneja la mayoría de los funcionarios, pareciera que su núcleo es la pobreza, de la cual se deriva el resto de los "problemas psicosociales". De esta manera, la inclusión de esta noción, más que traducirse en un rescate de la subjetividad del paciente, ha significado que se divulgue una relación casi directa entre el fenómeno de la policonsulta y la pobreza, lo cual resulta por lo menos cuestionable, pues responde a una ecuación bastante básica y que prioriza un tipo de realidad social por sobre las subjetividades implicadas. De alguna manera, se vuelve, con otras palabras, a la noción de pauperismo, tan cara a la tradición higienista. Y, de paso, se acepta una psicología simplificada de y para los pobres, asumiendo que su densidad subjetiva es tan mínima que no es diferenciable de las condiciones objetivas de existencia.

\section{Para finalizar}

El esfuerzo que realiza la medicina biológica por comprender - o más bien por acallar- las quejas corporales de los policonsultantes resulta infecundo, y estas insisten como un síntoma doloroso ante la mirada impotente de los profesionales de la salud. Dichos pacientes demandan que se les resuelva un problema en el plano corporal y la medicina, al asumir esta solicitud desde su literalidad, considera que no hay nada que hacer al respecto, ya que los instrumentos diagnósticos demuestran empíricamente que no hay una molestia real que requiera ser tratada. A partir de esto se rechaza la solicitud de estos pacientes, excluyendo cualquier posibilidad de reflexión respecto de los aspectos subjetivos que pudieran estar implicados en la consulta reiterada que presentan. Sin embargo, el paciente policonsultante reacciona frente a la falta de respuesta del sistema y se rehúsa a permanecer en el lugar de exclusión al que es enviado por la institución de salud. Lucha con insistencia por ser reconocido, quejándose para ser admitido como paciente.

El fenómeno de la policonsulta evidencia las debilidades del enfoque biomédico, ya que pone en jaque el método que la medicina occidental ha desarrollado en los últimos doscientos ańos para constituirse como disciplina científica. En definitiva, los pacientes policonsultantes desafían la construcción biológica del cuerpo, la cual, si bien ha permitido avances enormes en el alivio, la curación de enfermedades y la prolongación de la vida, margina y desconoce cotidianamente una serie de males y dolencias. La insistencia de los pacientes policonsultantes muestra que, a pesar de los intentos por introducir una mirada más integral dentro del Sistema de Salud, aún prevalece un enfoque que reduce al ser humano a su funcionamiento biomecánico y bioquímico, ofreciendo una respuesta solo a quienes logran ser diagnosticados con algún rótulo conocido. Esto se agrava, además, en la medida en que lo servicios públicos tienden a organizarse en torno a la oferta y no en torno a la demanda, teniendo que cumplir metas de gestión.

Si los pacientes policonsultantes reclaman reconocimiento, hay que recordar que este solo puede administrarse a través de un arte capaz de auscultar y maniobrar en las sutilezas más profundas de las relaciones humanas. Por supuesto, cabe preguntarse qué corresponde hacer y no hacer, y cuáles son los límites de cualquier establecimiento o profesional de la salud frente a este tipo de demandas. Ciertamente, hay formas paternalistas de responder a esta situación, que van invalidando, infantilizando a los pacientes. ¿Pero acaso no corresponde a las instituciones sanitarias ser a lo menos conscientes de su protagonismo social y del valor simbólico de todo aquello que concierne a la salud en nuestra sociedad? Resulta central detenerse en este punto, pues hoy se trata de demandas de atención canalizadas a través del Sistema de Salud, las cuales, al no cumplir con ciertos estándares, son excluidas. Sin embargo, siempre existe la tentación de medicalizar estas demandas, creando nuevos rótulos que de manera renovada desconozcan lo que está en juego. Después de todo, la violencia del diagnóstico médico no solo se ejerce sobre quienes reciben una etiqueta a la cual se identifican, sino también sobre aquellos que carecen de esta, lo que incluso puede tener mayores repercusiones subjetivas; "...en una sociedad morbosa predomina la creencia de que la mala salud definida y diagnosticada es infinitamente preferible a toda otra forma de etiqueta negativa o a la falta de toda etiqueta" (22:158). Es decir, la respuesta no está en una benevolencia que puede llegar a ser negligente, pero tampoco 
en abultar los manuales diagnósticos con nuevas categorías. Por el contrario, se trata de ejercer una clínica capaz de reconocer los aspectos imaginarios y simbólicos de la corporalidad, y preparada para hacer frente a los elementos intersubjetivos de la relación médico-paciente.

Si los pacientes policonsultantes se presentan ante los servicios de salud con una dolencia física, lo que buscan puede ser no más que la posibilidad de una mediación que permita simbolizar una experiencia corporal. Por eso no son dóciles, porque la experiencia implica una apropiación de la palabra del médico para construir sentido sobre el malestar que los aqueja(5). Eso hace necesario desplegar una clínica que no puede ser estándar ni practicada en serie.

\section{Agradecimientos}

Los autores agradecen al Centro de Salud Familiar Cardenal Raúl Silva Henríquez, de la comuna de Peñalolén, por la posibilidad de entrevistar a pacientes y funcionarios. 


\section{Referencias}

1. Nemours Foundation. Words! A Glossary of Medical Words (serial online) 1995-2013 (citado 25 de diciembre 2012). Disponible en: http://websrv02.kidshealth.org/kid/word/\#khsc

2. Picado L. La policonsulta y su impacto en los costos de los EBAIS concentrados del área de salud Alajuela sur durante el año 2001. Tesis de Maestría en administración de servicios de salud sostenibles. Costa Rica: Universidad Estatal a distancia; 2002.

3. Chile, Ministerio de Salud, Servicio de Salud Metropolitano Norte. Policonsulta y Servicio de Urgencia Adultos; 2005.

4. Velasco S, Ruiz MT, Alvarez-Dardet C. Modelos de atención a los síntomas somáticos sin causa orgánica: De los trastornos fisiopatológicos al malestar de las mujeres. Rev Esp Salud Pública 2006; 80 (4): 317-333.

5. Fagalde M, Guajardo G, Izquierdo D, Ayala B, Rojas P, Rojas S. Usuarias policonsultantes del nivel primario del Servicio de Salud Metropolitano Oriente: los sentidos de la enfermedad. Objetos Caídos 2012; 1 (citado el 22 de diciembre de 2012) Disponible en: http://www.academia.edu/1173416/Usuarias_policonsultantes_del_nivel_primario_de_atencion_del_Servicio_de_Salud_Metropolitano_Oriente_los_sentidos_de_la_enfermedad

6. Di Silvestre C. Somatización y percepción subjetiva de la enfermedad. Cinta de Moebio, Facultad Ciencias Sociales, Universidad de Chile 1998 (citado el 2 de enero de 2013); Disponible en: http://www2.facso.uchile.cl/publicaciones/ moebio/04/silvestre.htm

7. Le Breton D. Antropología del Cuerpo y Modernidad. Buenos aires: Nueva visión; 2002.

8. Saffie X. Pacientes (poli)consultantes en la Atención Primaria. Análisis de la policonsulta en un Centro de Salud Familiar desde la teoría psicoanalitica. Tesis para otra al grado de Magíster en Psicología Clínica. Universidad de Chile; 2012

9. Laplantine F. Antropología de la Enfermedad. Buenos Aires: Ediciones del Sol; 1999.

10. Schade N, Gonzáles A, Beyebach M, Torres P. Trastornos somatomorfos en la atención primaria: características psicosociales y resultados de una propuesta de consejería familiar. Rev Chil Neuro-Psiquiat 2010; 48(1): 20-28.

11. Thomas P. Determinantes psicosociales del fenómenos de la policonsulta. Revista Costarricense de Ciencias Médicas 1981; 2(2): 60-69.

12. Kottow M, Bustos R. Antropología Médica. Santiago: Editorial Mediterráneo; 2005.

13. Foucault M. El Nacimiento de la Clínica: una arqueología de la mirada médica. Buenos Aires: Siglo XXI; 2003.

14. Galende E. Psicoanálisis y Salud Mental. Para una crítica de la razón psiquiátrica. Buenos Aires: Paidós; 1990.

15. Foucault M. Los Anormales. Madrid: Ediciones Akal; 2001.

16. Maldonado V. El cambio social: políticas públicas 1990-2008. En: Bascuñán C, Correa G, Maldonado J, Sánchez V, (eds.) Más acá de los sueños, más allá de lo posible: la Concertación en Chile, Volumen II. Santiago: LOM Ediciones; 2009: $9-42$.

17. Sojo A. Reformas de Gestión en la Salud Pública en Chile. Chile, Serie Políticas Sociales (Revista en la Internet), Comisión Económica para América Latina y el Caribe (CEPAL); marzo 1996 (citado el 22 de junio de 2012); 13. Disponible en: http://www.eclac.org/cgi-bin/getProd.asp?xml=/publicaciones/xml/6/15216/P15216.xml\&xsl=/dds/tpl/p9f. xsl\&base=/dds/tpl/top-bottom.xsl

18. Borrell F. El modelo biopsicosocial en evolución. Med Clin 2002; 119: 175-179.

19. Gobierno de Chile, Ministerio de Salud Chile. Manual de apoyo a la implementación del modelo de atención integral con enfoque familiar y comunitario en establecimientos de atención primaria. Santiago de Chile: MINSAL; 2008.

20. Román O, Muñoz F. Una mirada crítica en torno al plan AUGE. Algunos aspectos generales y valóricos. Revista Médica de Chile 2008; 136(12): 1599-1603.

21. Irizar L. La cuestión de la subjetividad y la enfermedad. Versión abreviada Tesis Doctoral (versión online). 15 julio 2009 (citada el 21 de junio de 2012) Disponible en: http://psicoanalisisymedicina.blogspot.com/2009/07/la-cuestion-de-lasubjetividad-y-la.html

22. Illich I. Némesis Médica. México: Editorial Joaquín Mortiz; 1978.

Recibido: 30 de enero de 2013

Aceptado: 30 de abril de 2013 